


\section{What does the respiratory physician interested in sleep need to know about bariatric surgery?}

\section{Educational aims}

To understand the relationship between obstructive sleep apnoea/hypopnoea syndrome,
obesity and bariatric surgery.
To learn the mechanisms, techniques and non-respiratory benefits of bariatric surgery.

\section{Summary}

Obstructive sleep apnoea/hypopnoea syndrome (OSAHS) is reported to affect $4 \%$ of middle-aged males and $2 \%$ of middle-aged females in the UK [1], with one of the most widely recognised major risk factors being obesity [2]. The prevalence of obesity in many countries is rising - in England, $24 \%$ of males and 24\% of females were classified as obese in 2007 (body mass index (BMI) $\geq 30 \mathrm{~kg} \mathrm{per} \mathrm{m}^{2}$ ), compared with $13.2 \%$ of males and $16.4 \%$ of females in 1993 [3]. The prevalence of OSAHS within the obese population is widely reported to range between $55-90 \%$ in people with a BMl of $>40 \mathrm{~kg} \mathrm{per} \mathrm{m}^{2}$ [4], and many studies have found a reduction in the apnoea/hypopnoea index (AHI) and symptoms of OSAHS with weight loss [5], although it is widely recognised that weight loss by medical methods does not yield good results in the long term. The mainstay of treatment for OSAHS is nocturnal continuous positive airway pressure (CPAP) therapy. This has been shown to have a discontinuation rate of $18-24 \%$ [2], which adds to the need for alternative therapy for OSAHS associated with obesity.

Bariatric surgery is the field of weight-loss surgery, from the Greek words 'baros' meaning 'weight' and 'iatrike' meaning 'medicine', and is widely accepted as the most effective, long-term treatment for obesity, especially in those with a BMI of $>40 \mathrm{~kg}$ per $\mathrm{m}^{2}$. The Swedish Obese Subjects Trial, the largest trial studying surgical versus medical treatment of weight loss, showed an average weight loss of approximately $25.6 \% 2$ yrs after surgery and $18.3 \%$ after 10 yrs, compared with a weight change of $\pm 2 \%$ in the medically managed group [6].

\section{Entitlement}

The UK National Institute for Health and Clinical Excellence (NICE), the US National Institutes for Health and the International Federation for the Surgery of Obesity guidelines advise that bariatric surgery should be considered as a treatment option in obese patients who fulfil all of the following criteria [7].

1) $A$ BMl of $>40 \mathrm{~kg}$ per $\mathrm{m}^{2}$ or between $35-40$ $\mathrm{kg}$ per $\mathrm{m}^{2}$ and other significant disease, e.g. type 2 diabetes or hypertension, that could be improved if they lost weight.
H. Ramsey ${ }^{1}$

D.J. Pournaras ${ }^{2}$

A. Ahmed $^{2}$

C. le Roux ${ }^{2}$

M.R. Partridge ${ }^{1}$

${ }^{1}$ Dept of Respiratory Medicine, and ${ }^{2}$ Imperial Weight Centre, Imperial College Healthcare NHS Trust, Charing Cross Hospital, London, UK

\section{Correspondence}

H. Ramsey

Dept of Respiratory Medicine Charing Cross Hospital Fulham Palace Road London

SW6 8RF

UK

helen.ramsey@nhs.net

\section{Provenance}

Commissioned article, peer reviewed.

\section{Competing interests}

A. Ahmed has received educational grants from Ethicon and Covidien for attending and organising courses on bariatric surgery. 
2) All appropriate non-surgical measures have been tried but have failed to achieve or maintain adequate, clinically beneficial weight loss for at least 6 months.

3) The patients have received or will receive intensive management in a specialist obesity service.

4) The patients are generally fit for anaesthesia and surgery.

5) The patients are committed to the need for long-term follow-up.

The NICE guidance also recommends bariatric surgery as a first-line option in patients with a BMI of $>50 \mathrm{~kg}$ per $\mathrm{m}^{2}$ in whom surgery is deemed appropriate.
Figure 1

A Roux-en-Y gastric bypass procedure. Illustration courtesy of A.R. Ahmed.

\section{Figure 2}

The laparoscopic adjustable gastric band procedure. Illustration courtesy of A.R. Ahmed.

\section{Figure 3}

The sleeve gastrectomy procedure. Illustration courtesy of A.R. Ahmed.
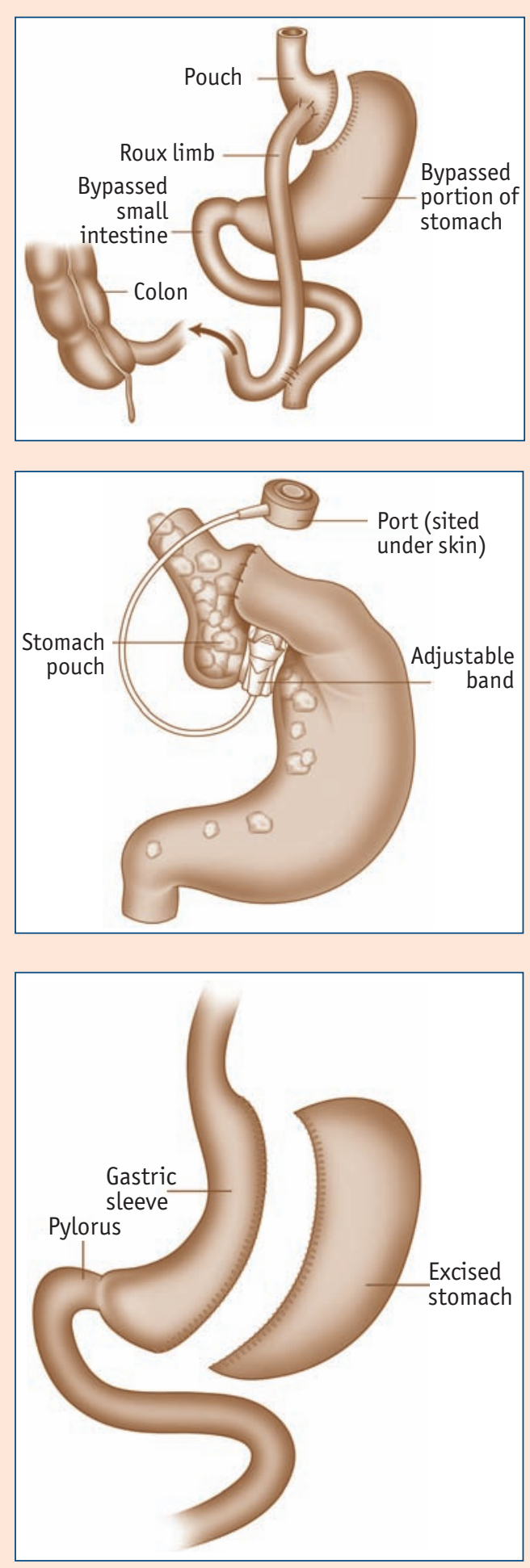

\section{Techniques of bariatric surgery}

Bariatric surgery works via many mechanisms, some of which are poorly understood. Historically it was separated into either restrictive or malabsorptive techniques, or a combination of both. However, there is probably no role played by malabsorption, as malabsorption of calories or proteins has not been shown to occur following most of the procedures. The most common procedure worldwide is the Rouxen-Y gastric bypass, which was initially developed in the 1960s. This procedure involves the formation of a small $(<30 \mathrm{~mL})$ stomach pouch that drains food into an alimentary or Roux limb (figure 1), whilst the remainder of the stomach secretes gastric acid, pepsin and intrinsic factor into a biliopancreatic limb which combines with pancreatic enzymes and bile and joins the alimentary (Roux) limb approximately $100 \mathrm{~cm}$ from the gastrojejunostomy. This procedure shortens the small intestine but still allows digestion and absorption. Roux-en-Y bypass works by reducing appetite in association with increased concentration of satiety gut hormones [8]. Sustained weight loss has been reported up to 16 yrs postoperatively with gastric bypass, and an average excess weight loss of $50-75 \%$ has been seen $1-2$ yrs postoperatively [9], or a total weight loss of $25-30 \%$. The second most common procedure is laparoscopic adjustable gastric banding (LACB) (figure 2), which restricts the stomach by the placement of an adjustable band below the gastro-oesophageal junction, and the use of a port placed subcutaneously through which saline is injected allowing for adjustment of the band. The LAGB works by reducing hunger allowing patients to consume fewer calories per day. Other procedures include sleeve gastrectomy (figure 3), which has been shown to work through reduced hunger, increased speed of gastric emptying and alterations in gut hormones [10]. Procedures that do have a malabsorptive component include the biliopancreatic diversion and duodenal switch [11].

The advent of improved laparoscopic techniques in recent years has reduced post-operative pain, shortened hospital stays and decreased rates of wound infection and hernia formation [12]. 


\section{Complications in the OSAHS population}

The complications of bariatric surgery differ depending on the nature of the procedure, but in one meta-analysis the overall mortality of all patients was found to be $<0.3 \%$ [13], which is similar to laparoscopic cholecystectomy. In patients undergoing bariatric surgery, those with OSAHS have an increased length of stay and higher cost for postoperative care [14]. Patients with OSAHS also have higher surgical risk due to worsening of obstructive events with anaesthetic and analgesic medications, as well as higher rates of postoperative cardiac arrhythmias, myocardial ischaemia, haemodynamic compromise and respiratory failure. These are most prevalent during the first $24 \mathrm{~h}$ after surgery [15]. The Longitudinal Assessment of Bariatric Surgery (LABS) consortium studied 4,776 patients who had undergone bariatric surgery (Roux-en-Y bypass or LAGB) and found a 30-day rate of death of $0.3 \%$, with $4.3 \%$ of patients having one or more major adverse event. OSAHS, a previous history of deep vein thrombosis or pulmonary embolus, or impaired functional status were independent risk factors for death at 30 days [16].

\section{Non-respiratory benefits}

Bariatric surgery aims to reduce the healthcare implications associated with obesity, whilst also having the added benefit of improving quality of life and reducing overall medication costs for patients. Various meta-analyses have shown a reduction in morbidity from other conditions that are more prevalent in obesity, for example diabetes, hyperlipidaemia and hypertension [17]. A recent meta-analysis of bariatric surgery showed that out of 135,246 patients, those with diabetes had a resolution of diabetes of $78.1 \%$ and $86.6 \%$ had either resolution or improvement in diabetes [18]. An earlier meta-analysis by the same group showed [19]: 1) an improvement in hyperlipidaemia in $\geq 70 \%$ of patients; 2 ) resolution of hypertension in $62 \%$ and improvement in 79\%; and 3) an improvement in symptoms of gastro-oesophageal reflux disease.

\section{Benefits in OSAHS}

Many studies have reported an improvement in OSAHS following bariatric surgery, although most are small studies with a lack of long-term follow-up and postoperative polysomnography. A recent meta-analysis of 12 studies, representing 342 patients, investigating the effects of surgical weight loss on obstructive sleep apnoea (OSA) showed that bariatric surgery significantly reduces the AHI but that the AHI after surgery was still consistent with moderate OSA, suggesting that patients undergoing bariatric surgery should not expect a cure for OSA after surgical weight loss [20]. However, several studies have recognised that daytime sleepiness is commonly associated with obesity in the absence of OSA or other sleepdisordered breathing [21, 22]; therefore, improvement in obesity, as well as an improvement in $\mathrm{AHI}$, may result in patients no longer meeting the diagnostic criteria for OSAHS. There have also been reports of recurrence of OSAHS in patients who have had bariatric surgery 7.5 yrs previously, without any increase in weight [23], confirming that although obesity is a major risk factor for OSAHS it is not the only risk factor.

Other studies into OSAHS and weight loss have shown a reduction in apnoeas and hypopnoeas in the order of $25 \%$ for every $10 \%$ of body weight lost [5], which has been suggested to be due to reduction in mass loads in the airway from surrounding adipose tissue, restoration of the tracheal tug on pharyngeal tissues and improved neuromuscular control: all of which decrease the pressure needed to keep the airway open [24]. The same article also reports a reduction in CPAP pressure requirement seen after bariatric surgery, with a reduction in median pressure of $16 \mathrm{CmH}_{2} \mathrm{O}$ at surgery to $8 \mathrm{cmH}_{2} \mathrm{O}$ at 7-months follow-up [24]. In patients without underlying obesity hypoventilation or chronic obstructive pulmonary disease, the authors have suggested that autotitrating CPAP should, therefore, be used in bariatric patients postoperatively to allow for a reduction in CPAP pressure [24].

\section{Bariatric surgery and asthma}

Interestingly, in obese patients with asthma several studies have shown that following bariatric surgery $90 \%$ of patients showed a reduction in symptoms of asthma 2 yrs after surgery, with $50 \%$ of patients having complete remission [25]. However, there is concern that the diagnosis of asthma may not be correct in some obese patients.

\section{Conclusions}

It is widely recognised that obesity is a risk factor for OSAHS and that OSAHS and obesity both

\section{Educational questions}

Which statement is correct?

1. With regards to obesity:

a) Prevalence has increased by $3 \%$ in 14 yrs in the UK for males

b) A person is classified as obese if they have a BMI of $\geq 30 \mathrm{~kg}$ per $\mathrm{m}^{2}$

c) In the UK there was a higher percentage of obese females compared to males in 2007

2. With regards to OSAHS: a) It affects $10 \%$ of middleaged males

b) It is effectively treated with CPAP in all patients c) It is reported to affect between $55-90 \%$ of those with a $\mathrm{BMI} \geq 40 \mathrm{~kg}$ per $\mathrm{m}^{2}$

3. Bariatric surgery:

a) Is as successful as medical management in the long term

b) Can be performed on those with a BMI of 35-40 $\mathrm{kg}$ per $\mathrm{m}^{2}$ if they have other significant comorbidities c) Has a lower mortality rate in patients with OSAHS than in those without

4. Benefits of bariatric surgery include:

a) A reduction in $\mathrm{AHI}$ in the order of $25 \%$ for every $10 \%$ of body weight lost

b) $50 \%$ improvement in diabetes

c) $85 \%$ resolution of hypertension 
lead to increased mortality and morbidity. Weight loss improves OSAHS, but is difficult to achieve and maintain by diet alone. Bariatric surgery is the most effective method of long-term weight loss and has been shown to improve OSAHS and reduce CPAP pressures, but is only available for patients fulfilling certain criteria. Obese patients have been noted to suffer from daytime hypersomnolence in the absence of OSAHS, thus, an improvement in symptoms postoperatively is not always associated with an improvement in obstructive episodes at polysomnography. Further studies are warranted to assess this further and to assess the long-term morbidity associated with recurrent obstructive episodes in the absence of daytime symptoms. Randomised controlled trials are needed to compare bariatric surgery and best medical treatment in patients with OSAHS alone and, depending on these results, in the future bariatric surgery may be a treatment option for patients with OSAHS and a $\mathrm{BMI}<35 \mathrm{~kg}$ per $\mathrm{m}^{2}$ if further trials show benefit.

\section{References}

1. National Institute for Health and Clinical Excellence (NICE). Guidance on sleep apnoea and continuous positive airways pressure (CPAP). www.nice.org.uk/TA139 Date last updated: March 2008.

2. Santos M, Rocha N, Filho J, et al. Obstructive Sleep apnea-hypopnea syndrome: the role of bariatric and maxillofacial surgeries. Obes Surg 2009; 19:796-801.

3. Statistics on obesity, physical activity and diet: England February 2009. Health and Social Care Information Centre, 2009. www.ic.nhs.uk/webfiles/publications/opan09/0PAD\%20Feb\%202009\%20final.pdf

4. Lettieri CJ, Eliasson AH, Greenburg DL. Persistence of obstructive sleep apnoea after surgical weight loss. J Clin Sleep Medicine 2008; 4: 333-338.

5. Peppard PE, Young T, Palta M, et al. Longitudinal study of moderate weight change and sleep-disordered breathing. JAMA 2000; 284: 3015-3021.

6. Sjöström L, Narbro K, Sjöström D, et al. Effects of bariatric surgery on mortality in Swedish obese subjects. N Engl J Med 2007; 357: 8741-8752.

7. National Institute for Health and Clinical Excellence. Obesity: guidance on the prevention, identification, assessment and management of overweight and obesity in adults and children. NICE clinical guideline 43. National Collaborating Centre for Primary Care and the Centre for Public Health and Excellence at NICE, 2006. www.nice.org. uk/nicemedia/pdf/CG43NICEGuideline.pdf

8. le Roux CW, Welbourn R, Werling M, et al. Gut hormones as mediators of appetite and weight loss after Roux-en-Y gastric bypass. Ann Surg 2007; 246: 780-785.

9. MacLean LD, Rhode BM, Sampalis J, et al. Results of the surgical treatment of obesity. Am J Surg 1993; 165: 155.

10. Melissas J, Daskalakis M, Koukouraki S, et al. Sleeve gastrectomy; a 'food limiting' operation. Obes Surg 2008; 18: 1251-1256.

11. Pournaras DJ, le Roux CW. After bariatric surgery, what vitamins should be measured and what supplements should be given? Clin Endocrino 2009; 71: 322-325.

12. Mun EC, Tavakkolizadeh. Surgical Management of Severe Obesity. www.uptodate.com

13. Maggard, MA, Shugarman, LR, Suttorp, M, et al. Meta-analysis: surgical treatment of obesity. Ann Intern Med 2005; 142: 547.

14. Kaw $R$, Aboussouan $L$, Auckley $D$, et al. Challenges in pulmonary risk assessment and perioperative management in bariatric surgery patients. Obes Surg 2008; 18: 134-138.

15. Salord N, Mayos M, Miralda R, et al. Respiratory sleep disturbances in patients undergoing gastric bypass surgery and their relation to metabolic syndrome. Obes Surg 2009; 19: 74-79.

16. Longitudinal Assessment of Bariatric Surgery (LABS) consortium, Flum DR, Belle SH, et al. Perioperative safety of the longitudinal assessment of bariatric surgery. N Engl J Med 2009; 361: 445-451.

17. Mango VL, Frishman WH. Physiologic, psycholgic and metabolic consequences of bariatric surgery. Cardiol Rev 2006; 14: 232-237.

18. Buchwals H, Estok R, Fahrbach K, et al. Weight and type 2 diabetes after bariatric surgery: systematic review and meta-analysis. Am J Med 2009; 122: 248-256.

19. Buchwald H, Avidor Y, Braunwald $\mathrm{E}$, et al. Bariatric surgery: a systematic review and meta-analysis. JAMA 2004; 292: 1724.

20. Greenburg DL, Lettieri CJ, Eliasson AH. Effects of surgical weight loss on measures of obstructive sleep apnoea: a meta-analysis. Am J Med 2009; 122: 535-542.

2. Dixon JB, Dixon ME, Anderson ML, et al. Daytime sleepiness in the obese: not as simple as obstructive sleep apnoea. Obesity 2007; 15: 2504-2511.

22. Vgontzas AN, Bixler EO, Tan T-L, et al. Obesity without sleep apnoea is associated with daytime sleepiness. Arch Intern Med 1998; 158: 1333-1337.

23. Pillar G, Peled R, Lavie P. Recurrence of sleep apnoea without concomitant weight increase 7.5 years after weight reduction surgery. Chest 1994; 106: 1702-1704. 1993; 3: 15-21. 


\section{Further reading}

Dixon JB, Dixon ME, Anderson ML, et al. Daytime Sleepiness in the obese: not as simple as obstructive sleep apnoea. Obesity 2007; 15: 2504-2511.

A study of pre-operative patients showing daytime sleepiness is common in severe obesity and unrelated to obstructive sleep apnoea.

Sjöström L, Narbro K, Sjöström CD, et al. Effects of bariatric surgery on mortality in Swedish obese subjects. N EnglJ Med 2007; 357: 741-752.

Long-term results of bariatric surgery in the largest and longest trial to date. 